\title{
Effects of exercise-based prehabilitation in children
}

\section{undergoing elective surgeries: a systematic review [version 1;}

\section{peer review: 1 approved]}

\author{
Jean Noronha(iD), Stephen Samuel (iD)2, Vijay Pratap Singh (iD3, \\ H Shivananda Prabhu 4
}

\begin{abstract}
${ }^{1}$ Department of Physiotherapy, Kasturba Medical College, Mangalore, Manipal Academy of Higher Education, Manipal, India ${ }^{2}$ Department of Physiotherapy, Kasturba Medical College, Mangalore,, Manipal Academy of Higher Education, Manipal, India ${ }^{3}$ Department of Physiotherapy, Kasturba Medical College Mangalore, Manipal Academy of Higher Education, Manipal, India ${ }^{4}$ Department of Surgery, Kasturba Medical College, Mangalore, Manipal Academy of Higher Education, Manipal, India
\end{abstract}

V1 First published: 08 Dec 2021, 10:1262

https://doi.org/10.12688/f1000research.74493.1

Latest published: 10 Jan 2022, 10:1262

https://doi.org/10.12688/f1000research.74493.2

\section{Abstract}

Background: Prehabilitation is a therapeutic strategy involving preoperative physical exercises, nutritional support, and stress and anxiety reduction. This approach has been gaining popularity and has been seeing effective results in adults in terms of improving pre and postoperative outcomes. The purpose of this review was to summarise the evidence about the effects of exercise-based prehabilitation programs on various outcome measures in children post elective surgeries.

Methods: PubMed, Scopus, Web of Science, PEDro, CINAHL/EBSCO and EMBASE electronic databases were searched from inception to June 2021. Based on the inclusion criteria, titles and abstracts were independently screened by the authors. After that, a data extraction table of the selected studies which included the participants, type, and details of exercise intervention, outcome measures and results were analysed after which the quality assessment of the studies was done. Results: The search yielded 2219 articles of which three articles fulfilled the inclusion criteria with two studies being randomized controlled trials and one being a quasi-experimental pre-post type of study. One randomized controlled trial was on the effects of exercisebased prehabilitation in reducing pulmonary complications post cardiac surgeries in children and the other two studies were on the effects of prehabilitation on functional capacity \& pulmonary function. All the three articles found that exercise-based prehabilitation had a positive effect on children's post-surgery.

Conclusion: Although there is a paucity of evidence-based literature, we conclude based on the existing literature retrieved by our review that exercise-based prehabilitation improves postoperative outcomes and helps in reducing postoperative complications in children

\section{Open Peer Review}

Approval Status

1

2

version 2

(revision)

10 Jan 2022

version 1

08 Dec 2021

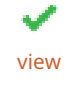

1. Forhad Akhtar Zaman , All India Institute of Medical Sciences (AIIMS), Guwahati, India

2. Anna-Maria Platschek, German Sport

University Cologne, Cologne, Germany

Any reports and responses or comments on the article can be found at the end of the article. 
undergoing various surgeries.

\section{Keywords}

Pre-operative exercises, preoperative exercise, prehabilitation, exercise therapy, surgery, paediatric, children, adolescents

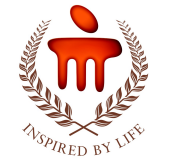

This article is included in the Manipal Academy

of Higher Education gateway.

Corresponding author: Stephen Samuel (stephen.samuel@manipal.edu)

Author roles: Noronha J: Conceptualization, Methodology, Writing - Original Draft Preparation, Writing - Review \& Editing; Samuel S: Conceptualization, Methodology, Writing - Original Draft Preparation; Singh VP: Conceptualization, Writing - Review \& Editing; Prabhu HS: Supervision, Writing - Review \& Editing

Competing interests: No competing interests were disclosed.

Grant information: The author(s) declared that no grants were involved in supporting this work.

Copyright: @ 2021 Noronha J et al. This is an open access article distributed under the terms of the Creative Commons Attribution License, which permits unrestricted use, distribution, and reproduction in any medium, provided the original work is properly cited.

How to cite this article: Noronha J, Samuel S, Singh VP and Prabhu HS. Effects of exercise-based prehabilitation in children undergoing elective surgeries: a systematic review [version 1; peer review: 1 approved] F1000Research 2021, 10:1262

https://doi.org/10.12688/f1000research.74493.1

First published: 08 Dec 2021, 10:1262 https://doi.org/10.12688/f1000research.74493.1 


\author{
Abbreviations used \\ ACBT: Active Cycle of Breathing Technique \\ CG: Control Group \\ FEV1: Forced Expiratory Volume 1 \\ FEV1/FVC Ratio: Tiffeneau-Pinelli index \\ FVC: Forced Vital Capacity \\ IG: Interventional group \\ PEFR: Peak expiratory flow rate \\ POP: Postoperative Physiotherapy \\ POPE: Preoperative Physiotherapy Education \\ PRISMA: Preferred Reporting Items for Systematic Reviews and Meta-Analyses \\ RCT: Randomized control trial \\ ROM: Range of Motion \\ TUGT: Time up and Go test \\ 6MWT: Six-minute walk test \\ 9SCT: 9 Step climbing test \\ 10MWT: 10 minute walk test
}

\title{
Introduction
}

Major surgeries in children along with the deleterious effects of the condition that predisposes a child for the surgery lead to complications that need to be therapeutically managed in children.

Prehabilitation is a multimodal type of approach that helps a patient planned for any major surgery and also allows them to prepare to, manage the stressors in the pre-surgical period and also undertake the necessary rehabilitation successfully so that they can return to their pre-operative state with better and improved outcomes. ${ }^{1,2}$ Prehabilitation encompasses preoperative physical exercises, nutritional support, and stress and anxiety reduction. ${ }^{3,4}$ The concept of prehabilitation dates as far back as World War II and was initially started not as a part of the pre-surgical procedure. ${ }^{1}$ Prehabilitation has its first mentions in articles in 1942 where it raised the fact that military recruits would be medically screened and treated in respect to their health and comorbidities, resulting in a higher number of acceptances. ${ }^{1,5}$ and came to light post-2011 after the systematic review published by Valkenet et al. about prehabilitation before joint, cardiac and abdominal surgeries ${ }^{3,6}$

Prehabilitation/preoperative exercise is a set of interventions done before surgery that helps the patient to be prepared for post-surgical stressors and also help improve their functional capacity (FC) through the exercises. ${ }^{1,7}$ Patients' ability to function to their fullest capacity can deteriorate because of inactivity during the surgical period and even if the surgery has been successful there can be chances of deconditioning. ${ }^{1,8,9}$ Current studies and reviews done in prehabilitation concerning the adult population does show that there is improvement in the post-operative complications and length of stay and also in their post-operative pain. ${ }^{3,10}$ Therefore the concept of prehabilitation is said to not only help the patient prepare themselves before a major surgery for post-surgical complications, but it also helps the patient to understand the importance of it to reduce the complications, helping them promote physical fitness and also optimize their psychological wellbeing. This also helps the patients return to their normal levels of functionality that was present before surgery. ${ }^{1}$

This review aims at examining the current body of evidence in the area of exercise-based prehabilitation in children undergoing various elective surgeries.

\section{Methods}

Search strategy

A data search was made on PubMed, Scopus, Web of Science, PEDro, EMBASE, CINAHL/EBSCO from inception to June 2021. The terms used for search for the paediatric population were the following: infant [Mesh], child [Mesh], adolescent [Mesh], children. The terms preoperative exercise [Mesh], exercise [Mesh], exercise therapy [Mesh], breathing exercises [Mesh], preoperative exercises [Mesh] were used related to the intervention and for the population type: general surgery [Mesh], paediatrics/surgery [Mesh] and surgical procedures operative [Mesh] were the terms used. The search terms were combined with a Boolean operator 'AND' or 'OR' wherever applicable. The references of the included articles were also screened for possible relevant studies.

\section{Selection criteria}

The articles were screened based on the following pre-set criteria. The inclusion criteria include 1) Studies that included participants in the age group of 0-18 years; 2) Studies that include children undergoing elective surgeries; 3 ) Studies published in English language and 4) were either randomized control trial (RCT), Non-RCT, single group post, case study 
and case series and the exclusion criteria included were 1) studies with participants undergoing a prehabilitation program other than exercise 2) studies that included participants above 18 years.

\section{Results}

Characteristics of studies

All the data retrieved from the databases, summing up to 2219 articles, were fed in the Mendeley Desktop v1.19.8 after which duplicates were removed. The articles were then screened through the titles and 181 articles were found eligible, following this the abstract screening removed 150 articles, after which full-text screening was done, and 29 papers were excluded, eventually yielding three papers that meet the inclusion criteria of this review. The PRISMA flow chart in Figure 1. Outlines details regarding the identification, screening, eligibility, and inclusion of the studies in this review.

\section{Qualitative analysis}

The risk of bias scoring was done using the NIH Quality assessment scales as shown in Figure $2 .{ }^{14}$ The quality assessment scales were used depending on the type of study. Two separate scales were used for the pre-post study design and the RCT. The scales covered everything regarding the type, duration of the study, the sample sizes, characteristics of the population and about its randomization, the interventions used, and whether participants and therapists were blinded. A score of 9/12 was rated for the pre-post type of study done by Sharma $\mathrm{N}$ et al. ${ }^{15}$

A score of 7/14 was given for the RCT done by Sharma N et al. ${ }^{16}$ and Felcar et al. ${ }^{17}$ respectively.

\section{Population of the included studies}

Of the three studies, the two studies included children scheduled for abdominal surgeries in the age group 5-17 years of age $^{15,16}$ and the other study had children one-day-old to six-year-old with congenital heart disease who underwent heart surgeries. $^{17}$

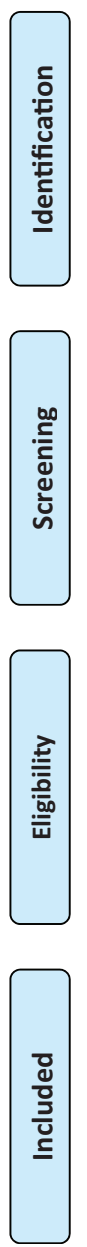

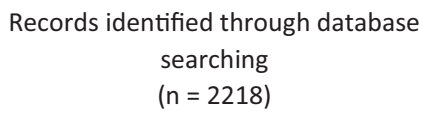

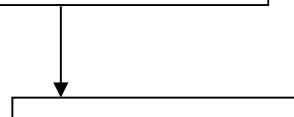

Records after duplicates removed $(n=2209)$

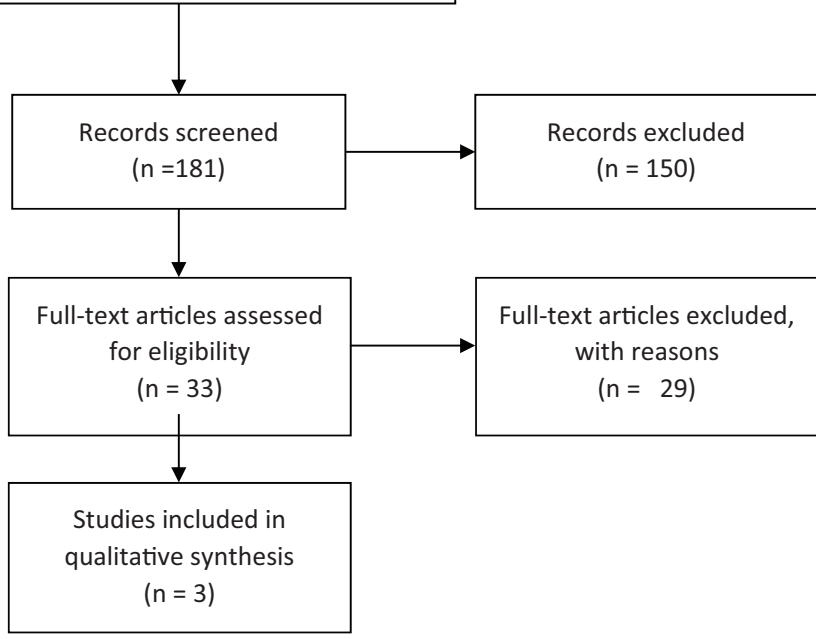

Figure 1. PRISMA flow chart. 
NIH - Study Quality Assessment Tools

Quality was rated as $0=$ poor (0-4 out of 14 questions)

$\mathrm{i}$ = for fair (5-10 out of 14 questions),

or ii = good (11-14 out of 14 questions);

NA: not applicable,

NR: not reported.

For Quasi-experimental pre-post study design

\begin{tabular}{|c|c|c|c|c|c|c|c|c|c|c|c|c|c|}
\hline $\begin{array}{l}\text { Question } \\
\text { no }\end{array}$ & 1 & 2 & 3 & 4 & 5 & 6 & 7 & 8 & 9 & 10 & 11 & 12 & \\
\hline $\begin{array}{l}\text { Neha } \\
\text { Sharma } \\
\text { et al }(15)\end{array}$ & Yes & Yes & Yes & Yes & Yes & Yes & Yes & NR & NA & Yes & Yes & NA & $\mathrm{i}$ \\
\hline
\end{tabular}

For RCTS

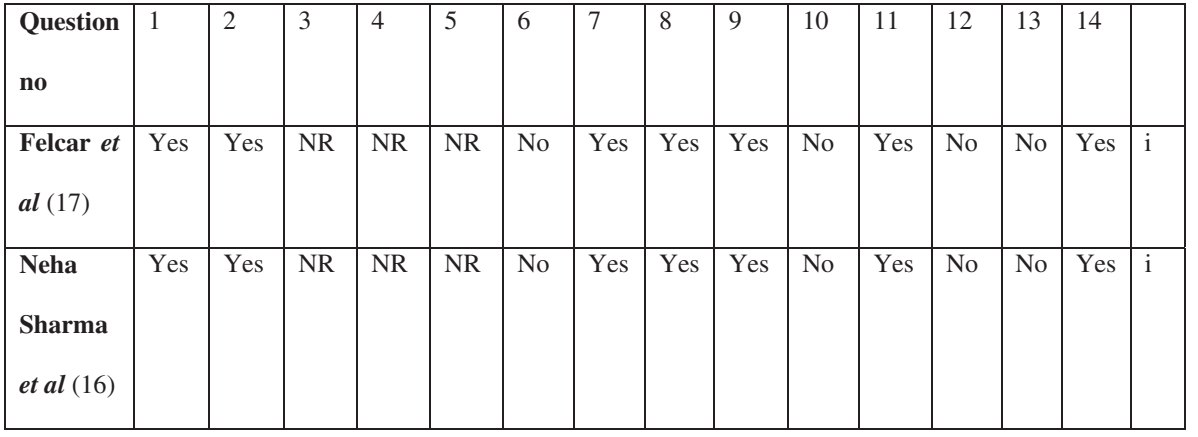

Figure 2. NIH study quality assessment tool. ${ }^{14}$

Intervention of the included studies

All of the three studies included exercise-based prehabilitation as the main form of intervention. Of the three studies, one study focused on the use of exercise-based prehabilitation in reducing pulmonary complications through chest physiotherapy, clearance techniques, support and guidance to parents, and early mobilization. ${ }^{17}$ The other two studies included the N-PARP protocol ${ }^{15}$ used for prehabilitation This protocol included exercises to be given from the pre-operative period till POD5 and included breathing exercises, ROM exercises, and ambulation. ${ }^{15,16}$

Outcomes of the included studies

Felcar et al. ${ }^{17}$ included the presence or absence of pulmonary complications as its major outcome measure. while Sharma $\mathrm{N}$ et al. ${ }^{15,16}$ had a pulmonary function and functional capacity (FC) as their main outcomes which included spirometer values and Six-minute walk test (6MWT) respectively and others being 10 minute walk test (10MWT), Timed up and go test (TUGT), chest expansion. A detailed explanation of the studies is given in Table 1.

\section{Data extraction}

A data extraction table was made to summarize and cover all the details regarding the participants, study design, sample size, study groups, type and dosage of exercise intervention, outcomes measures, and conclusion for all the selected studies. A detailed description of the Data extraction is presented in Table 1. 


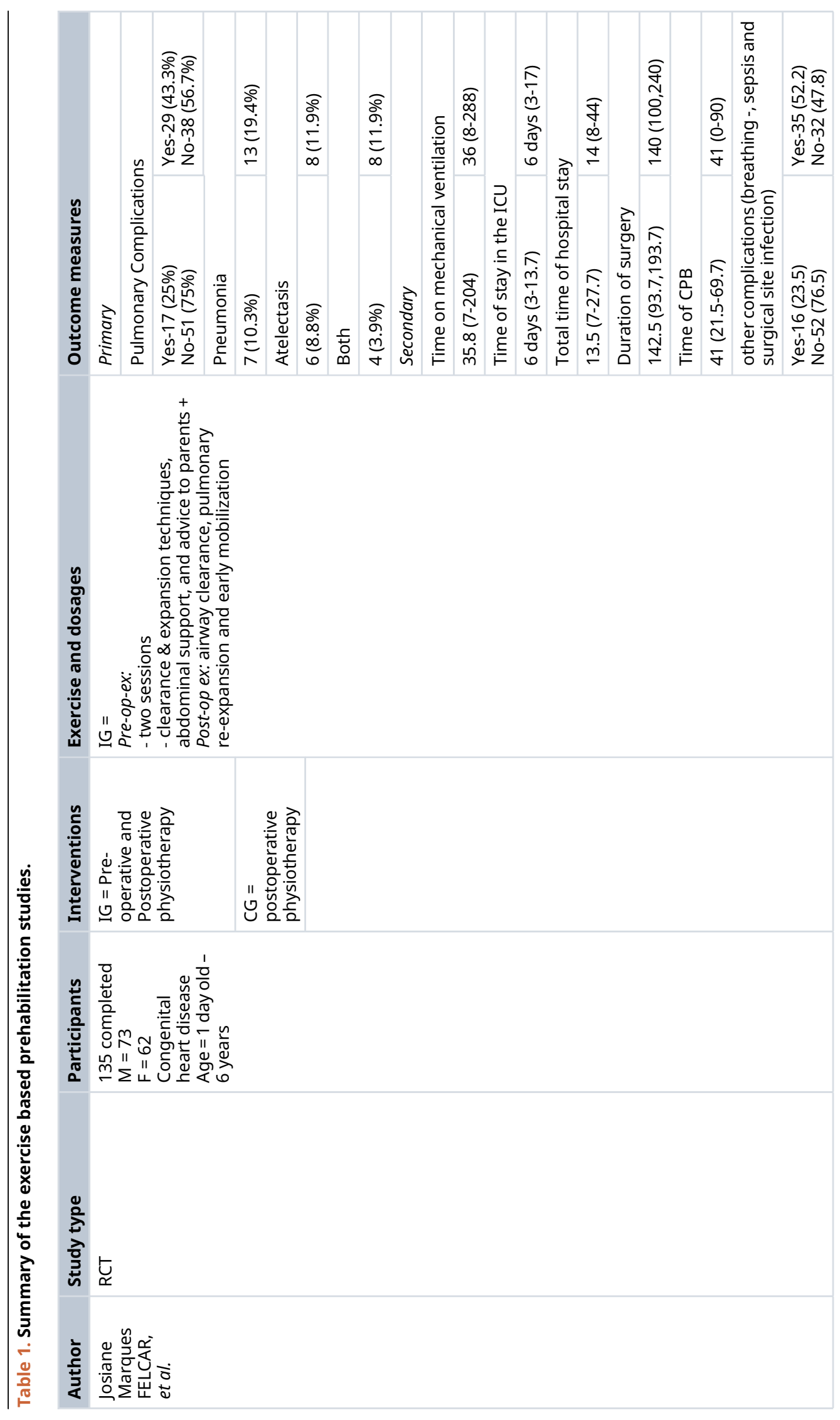




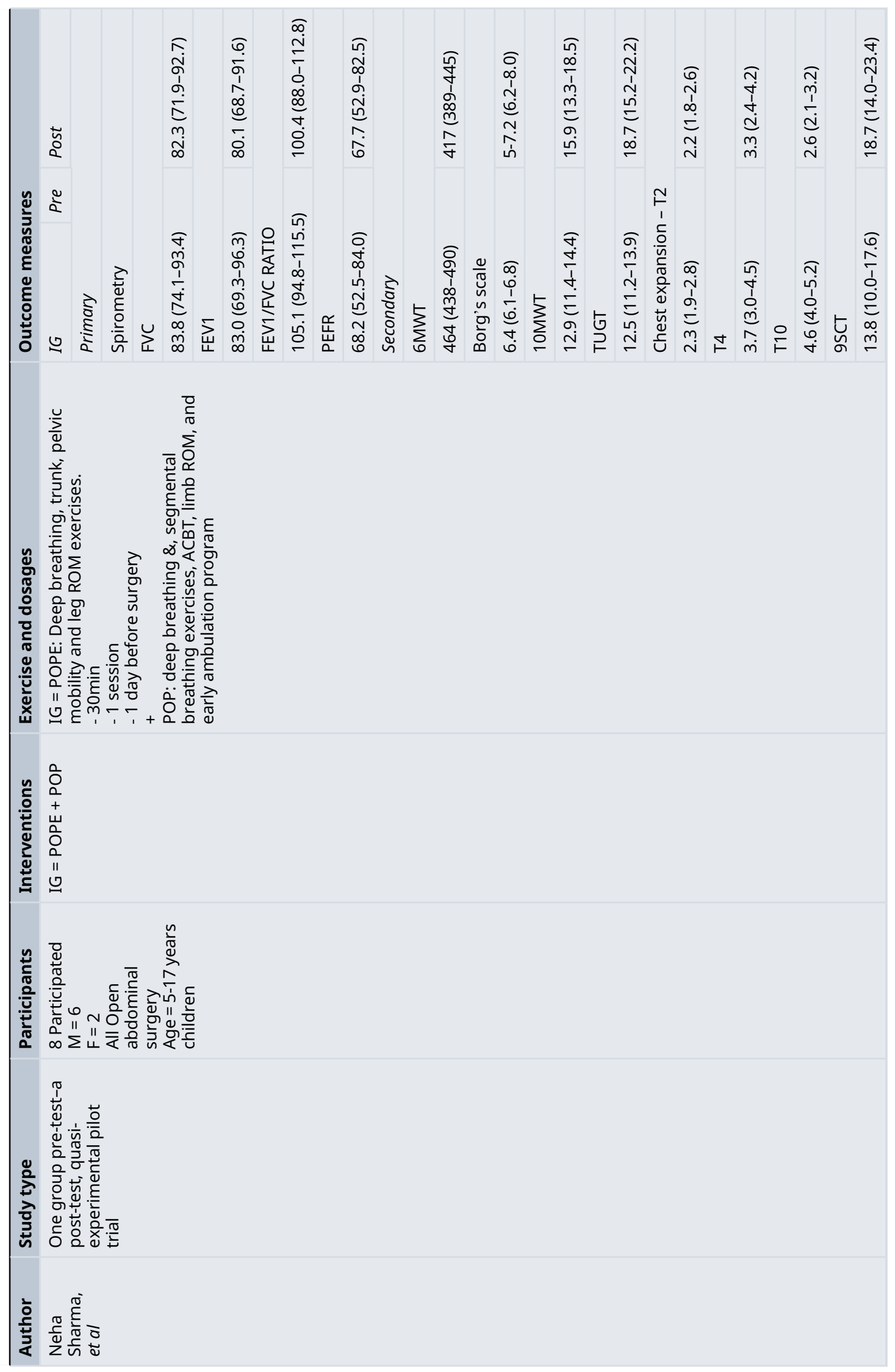




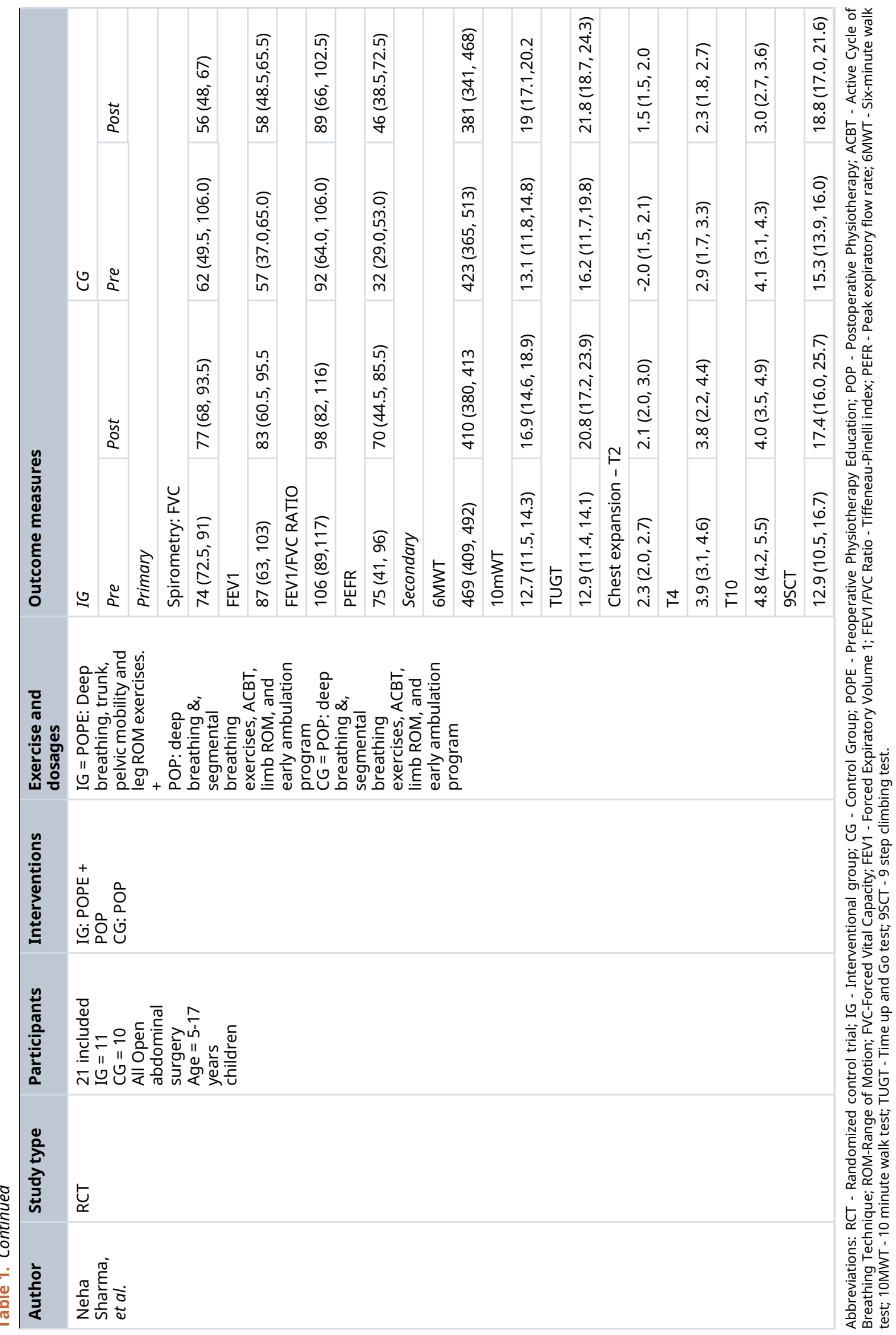




\section{Discussion}

This systematic review aimed at identifying studies that gave an exercise-based prehabilitation intervention to children undergoing various surgeries. While searching articles for this review various studies were found that included postoperative exercise after surgery in children, but very few studies included prehabilitation in the routine clinical care of these patients.

Sharma $\mathrm{N}$ et al. published two studies in $2020^{15}$ and in $2021^{16}$ about the effects of prehabilitation on pulmonary function and FC in the patients undergoing elective abdominal surgeries and Felcar $\mathrm{et}$ al. studied its effects in the reduction of postop pulmonary complications in children undergoing cardiac surgery. ${ }^{17}$

In the two studies conducted by Sharma $\mathrm{N}$ et al. ${ }^{15,16}$ the effects of prehabilitation on FC and pulmonary functions were studied using a spirometer as a measurement tool. There was a trend seen in both the studies that no major changes in the values of the spirometer (that includes Forced Vital Capacity (FVC), Forced Expiratory Volume 1 (FEV1), TiffeneauPinelli index (FEV1/FVC ratio), Peak Expiratory Flow Rate (PEFR)) were seen in the pre-surgical period and on POD 5, but there was a decline seen from the pre-operative period to POD 1 and from POD1 to POD5. The only difference seen in the $\mathrm{RCT}^{16}$ compared to the pre-post study ${ }^{15}$ conducted by Sharma N et al. was that FVC improved post prehabilitation and surgery. Values of chest expansion seemed to be better in the IG than CG in the RCT. ${ }^{16}$ Lastly, one of the common findings in both the studies was that the values of 10MWT, TUGT, 9SCT were seen better in the CG of both studies rather than in the IG. ${ }^{15,16}$ This study implies that exercise-based prehabilitation when given in a proper format and incorporated well in routine care can have beneficial effects in children during the post-operative period.

In the study done by Felcar et al., ${ }^{17}$ it was seen that children in the CG were seen to have a higher frequency of developing pulmonary complications such as pneumonia or atelectasis or both as compared to the IG that received both the treatment options, i.e., prehabilitation and post-operative exercises. ${ }^{17}$ This implies that children who received exercise-based prehabilitation have a lesser frequency of developing any other complications as compared to the children that didn't receive prehabilitation.

Quality assessment of each of the studies was done by using the NIH Quality assessment scale. Two different scales were used for each type of the study i.e. the pre-post type of study and the other for an $\mathrm{RCT}^{14}$ The Pre-post study was done by Neha et al. ${ }^{15}$ had a scoring of $9 / 12$ which acc to their scale was categorized as fair. This study included clearly stated objectives, had a pre-set inclusion and exclusion criteria. The sample size was around 12 participants but enough to conclude about the effects of the N-PARP (prehabilitation protocol) in that set population and to conclude that the study could be done in a larger population. there is also no information regarding the blinding of the populations in this study.

The NIH Quality assessment is done for The articles of Felcar et al., ${ }^{17}$ and Neha et al. ${ }^{16}$ scored them (7/14) and (7/14) respectively which according to their scale belonged to the fair category. Both these RCTs did not have any details regarding the blinding and concealment of the participants.

\section{Conclusion}

Although few in number, the available literature leads us to the conclusion that exercise-based prehabilitation plays an important role in improving health-related outcome measures in children undergoing various surgeries.

\section{Data availability}

Underlying data

All data underlying the results are available as part of the article and no additional source data are required.

Reporting guidelines

Open Science Framework: PRISMA Checklist final.docx, https://doi.org/10.17605/OSF.IO/B3CPX.

Data are available under the terms of the Creative Commons Attribution 4.0 International license (CC-BY 4.0). 
1. Wright $S$, Wiechula R, McLiesh P: The effectiveness of prehabilitation for adults having elective surgery: a systematic review protocol. JBI database Syst. Rev. Implement Reports. 2016; 14(2): 78-92.

PubMed Abstract | Publisher Full Text

2. Patel BK, Hall JB: Perioperative physiotherapy. Curr. Opin. Anaesthesiol. 2013; 26(2): 152-156.

PubMed Abstract | Publisher Full Text

3. Banugo P, Fcai M, Amoako D, et al.: Prehabilitation. 2017; 17(August): 401-5.

4. Ditmyer MM, Topp R, Pifer M: Prehabilitation in preparation for orthopaedic surgery. Orthop. Nurs. 2002; 21(5): 43-54. quiz 52. PubMed Abstract | Publisher Full Text

5. PREHABILITATION, rehabilitation, and revocation in the Army. Br. Med. J. 1946; 1: 192-197.

PubMed Abstract

6. Valkenet K, Van De Port IGL, Dronkers JJ, et al.: The effects of preoperative exercise therapy on postoperative outcome: A systematic review. Clin. Rehabil. 2011; 25(2): 99-111. Publisher Full Text

7. Kim DJ, Mayo NE, Carli F, et al.: Responsive measures to prehabilitation in patients undergoing bowel resection surgery. Tohoku J. Exp. Med. 2009; 217(2): 109-115. PubMed Abstract | Publisher Full Text

8. Pieper MLB: Perceptions of the waiting period before coronary artery bypass grafting. PubMed Abstract

9. Killewich LA: Strategies to minimize postoperative deconditioning in elderly surgical patients. J. Am. Coll. Surg. 2006; 203(5): 735-745.

PubMed Abstract | Publisher Full Text

10. Mina DS, Clarke $H$, Ritvo $P$, et al.: Effect of total-body prehabilitation on postoperative outcomes: a systematic review and meta-analysis. Physiotherapy. 2014; 100(3): 196-207. PubMed Abstract | Publisher Full Text

11. Cabilan CJ, Hines S, Munday J: The effectiveness of prehabilitation or preoperative exercise for surgical patients: a systematic review. JBI database Syst. Rev. Implement Reports. 2015; 13(1): 146-187.

Publisher Full Text

12. Topp $R$, Ditmyer $M$, King $K$, et al.: The effect of bed rest and potential of prehabilitation on patients in the intensive care unit. AACN Clin. Issues. 2002; 13(2): 263-276. PubMed Abstract | Publisher Full Text

13. Carli F, Zavorsky GS: Optimizing functional exercise capacity in the elderly surgical population. Curr. Opin. Clin. Nutr. Metab. Care. 2005; 8(1): 23-32.

PubMed Abstract | Publisher Full Text

14. Study Quality Assessment Tools|NHLBI, NIH: Reference Source

15. Sharma N, Sree BS, Aranha VP, et al.: Preserving pulmonary function and functional capacity in children undergoing open abdominal surgery: A one group pretest-posttest, quasiexperimental pilot trial. J. Pediatr. Surg. 2020; 55(10): 2191-2196. PubMed Abstract | Publisher Full Text

16. Sharma N, Sree BS, Samuel AJ, et al.: A randomized clinical trial in improving pulmonary function and functional capacity in pediatric open abdominal surgery. J. Pediatr. Surg. 2021 Mar [cited 2021 Apr 23]; 56(3): 559-564.

Publisher Full Text | PubMed Abstract | Reference Source

17. Felcar JM, Guitti JC d S, Marson AC, et al.: Preoperative physiotherapy in prevention of pulmonary complications in pediatric cardiac surgery. Rev. Bras. Cir. Cardiovasc. 2008; 23(3): 383-388.

PubMed Abstract | Publisher Full Text 


\section{Open Peer Review}

\section{Current Peer Review Status:}

\section{Version 1}

Reviewer Report 05 January 2022

https://doi.org/10.5256/f1000research.78256.r102366

(C) 2022 Zaman F. This is an open access peer review report distributed under the terms of the Creative Commons Attribution License, which permits unrestricted use, distribution, and reproduction in any medium, provided the original work is properly cited.

\section{Forhad Akhtar Zaman}

Department of Community Medicine \& Family Medicine, All India Institute of Medical Sciences (AIIMS), Guwahati, Assam, India

I think this is an excellent systematic review aimed at reviewing studies of exercise-based prehabilitation intervention to children undergoing various surgeries.

The strength of the study was that the review was done very meticulously and it followed all the required steps of systematic review. This will be an important addition to the existing literature. As the selection criteria was very robust \& specific, only three out of a whole lot of studies could be selected for the review. Although few in number, I really like the way in which the discussion was done and evidence presented. I hope the authors will correct the few numerical errors which which has been mentioned in the report.

I have only a couple of minor points of clarification:

1. In the Methodology section, it was not mentioned why only randomized controlled trials \& quasi-experimental studies were selected.

2. There is a mismatch between the number of total eligible titles (181) \& total removed (150), thus full-text articles assessed for eligibility should have been 31, but it is mentioned as 33 in the PRISMA Flow chart (Fig-1). Also, out of 33 full-text articles which was assessed for eligibility, 29 papers were excluded, leaving 4 papers that ultimately met the inclusion criteria, but it is mentioned as 3 .

Are the rationale for, and objectives of, the Systematic Review clearly stated? Yes

Are sufficient details of the methods and analysis provided to allow replication by others? Partly

Is the statistical analysis and its interpretation appropriate? 


\section{I cannot comment. A qualified statistician is required.}

Are the conclusions drawn adequately supported by the results presented in the review? Yes

Competing Interests: No competing interests were disclosed.

Reviewer Expertise: Communicable disease especially TB, HIV, Covid

I confirm that I have read this submission and believe that I have an appropriate level of expertise to confirm that it is of an acceptable scientific standard.

Author Response 06 Jan 2022

Stephen Samuel, Manipal Academy of Higher Education, Manipal, India

Dear F1000Research Team,

We thank the reviewer and the editorial team for taking out the time to conduct an in-depth review of our article. We thank you for the appreciation you have given us regarding this systematic review. We also thank you for the clarifications. The necessary changes have been made and addressed accordingly. The modified manuscript and the latest PRISMA flow chart with changes tracked in the word document have been uploaded.

\section{Reviewer Comment 1}

"In the Methodology section, it was not mentioned why only randomized controlled trials \& quasiexperimental studies were selected."

Reply - The following types of study designs, i.e., RCT (Randomized Controlled Trials), NonRCT, Single group Pre-post, Case study, and series were to be included; but during the data extraction and reviewing process only RCT and quasi-experimental studies were retrieved

\section{Reviewer Comment 2}

"There is a mismatch between the number of total eligible titles (181) \& total removed (150), thus full-text articles assessed for eligibility should have been 31, but it is mentioned as 33 in the PRISMA Flow chart (Fig-1). Also, out of 33 full-text articles which was assessed for eligibility, 29 papers were excluded, leaving 4 papers that ultimately met the inclusion criteria, but it is mentioned as 3."

Reply- We thank the reviewer for the correction and we have made the necessary changes as suggested.

Competing Interests: No competing interests were disclosed. 
The benefits of publishing with F1000Research:

- Your article is published within days, with no editorial bias

- You can publish traditional articles, null/negative results, case reports, data notes and more

- The peer review process is transparent and collaborative

- Your article is indexed in PubMed after passing peer review

- Dedicated customer support at every stage

For pre-submission enquiries, contact research@f1000.com 